\title{
Further evidence of nuclear reactions in the Pd/D lattice: emission of charged particles
}

\author{
Stanislaw Szpak • Pamela A. Mosier-Boss • \\ Frank E. Gordon
}

Published online: 18 April 2007

(C) Springer-Verlag 2007

\section{Erratum to: Naturwissenschaften}

\section{DOI 10.1007/s00114-007-0221-7}

In our above-mentioned paper, we describe a model that results in the generation of low-energy neutrons. We find that Widom and Larsen have done a thorough mathematical treatment that describes one mechanism to create such lowenergy neutrons. This mathematical treatment has been described in the following reference:

Widom A, Larsen L (2006) Ultra low momentum neutron catalyzed nuclear reactions on metallic hydride surfaces. Eur Phys J C46:107-111.

We regret the omission of this reference.

The online version of the original article can be found at http://dx.doi. org/10.1007/s00114-007-0221-7

S. Szpak $\cdot$ P. A. Mosier-Boss $(\square) \cdot$ F. E. Gordon Space and Naval Warfare (SPAWAR) Systems Center San Diego,

San Diego, CA 92152-5001, USA

e-mail: pam.boss@navy.mil 